\title{
Methyl cyanide as tracer of bow shocks in L1157-B1
}

\author{
C. Codella ${ }^{1}$, M. Benedettini ${ }^{2}$, M. T. Beltrán ${ }^{1}$, F. Gueth ${ }^{3}$, S. Viti ${ }^{4}$, R. Bachiller ${ }^{5}$, M. Tafalla ${ }^{5}$, S. Cabrit $^{6}$, \\ A. Fuente ${ }^{5}$, and B. Lefloch ${ }^{7}$
}

1 INAF - Osservatorio Astrofisico di Arcetri, Largo E. Fermi 5, 50125 Firenze, Italy e-mail: codella@arcetri.astro.it

2 INAF, Istituto di Fisica dello Spazio Interplanetario, via Fosso del Cavaliere 100, 00133 Roma, Italy

3 IRAM, 300 rue de la Piscine, 38406 Saint-Martin-d'Hères, France

4 Department of Physics and Astronomy, University College London, Gower Street, London WC1E6BT, UK

5 Observatorio Astrónomico Nacional (IGN), Alfonso XII, 28014 Madrid, Spain

${ }^{6}$ LERMA, UMR 8112 du CNRS, Observatoire de Paris, 61 Av. de l'Observatoire, 75014 Paris, France

7 Laboratoire d'Astrophysique de l'Observatoire de Grenoble, BP 53, 38041 Grenoble Cedex, France

Received 22 September 2009 / Accepted 20 October 2009

\section{ABSTRACT}

\begin{abstract}
Context. L1157-mm is a low-mass protostar driving an outflow which is considered the proto-type of the so-called chemically active outflows. In particular, the blue-shifted lobe B1 stands out for its rich mm-wave spectrum.

Aims. Our aim is to infer the physical conditions of the molecular gas within L1157-B1.

Methods. We carried out $\mathrm{CH}_{3} \mathrm{CN}\left(8_{K}-7_{K}\right)$ observations at $2 \mathrm{~mm}$ with the IRAM Plateau de Bure Interferometer to map L1157-B1. Results. The $\mathrm{CH}_{3} \mathrm{CN}$ image shows a clumpy structure superimposed to the classical $\mathrm{B} 1$ arch-like shape, displaying a unique continuous structure tracing the propagation of a large bow shock. The $\mathrm{CH}_{3} \mathrm{CN}$ spatial distribution well agrees with that of $\mathrm{CH}_{3} \mathrm{OH}$, one of the most abundant species evaporated from dust grain mantles. Thanks to the present $\mathrm{CH}_{3} \mathrm{CN}$ observations at unprecedented spatial resolution, we determine for the first time the temperature distribution of the B1 bow shock. We obtain values between 57 and $132 \mathrm{~K}$, with the highest temperature at the shock apex, thus confirming the association of $\mathrm{CH}_{3} \mathrm{CN}$ with gas affected by the passage of a shock wave. The $N_{\mathrm{CH}_{3} \mathrm{CN}}$ column densities are $\simeq 8-40 \times 10^{12} \mathrm{~cm}^{-2}$, depending on the position, leading to $\mathrm{CH}_{3} \mathrm{CN} / \mathrm{CH}_{3} \mathrm{OH}$ abundance ratios $\simeq 0.2-1.3 \times 10^{-3}$, i.e. values on average smaller than those found in hot-corinos. This suggests that a significant amount of $\mathrm{CH}_{3} \mathrm{CN}$ is formed in the gas phase: in the shocked L1157-B1 regions the $\mathrm{CH}_{3} \mathrm{CN}$ abundance may not have reached its maximum yet.
\end{abstract}

Key words. ISM: individual objects: L1157 - ISM: molecules - stars: formation

\section{Introduction}

During the earliest evolutionary stages, young stellar objects (YSOs) produce high-velocity jets which drive shocks travelling through the surrounding high-density medium. Jets compress, and with time, the ambient medium creates slower molecular outflows formed by swept-up material. Shocks trigger several processes such as endothermic chemical reactions and ice grain mantle sublimation, which lead to a dramatic enhancement of the abundance of several species (see e.g. van Dishoeck \& Blake 1998, and references therein). This has been demonstrated by multiline observations at millimeter wavelengths towards a number of outflows (e.g. Garay et al. 1998; Bachiller \& Pérez Gutiérrez 1999; Jörgensen et al. 2007). In particular, the L1157 outflow, powered by IRAS 20386+6751, may be regarded as the typical laboratory to observe the effects of shocks on the gas chemistry (Bachiller et al. 2001). IRAS 20386+6751 is a low-luminosity $\left(11 L_{\odot}\right)$ class 0 protostar, located at $440 \mathrm{pc}$ driving a precessing powerful molecular outflow that shows several bow shocks seen in CO (Gueth et al. 1996) as well as in NIR $\mathrm{H}_{2}$ images (Davis \& Eislöffel 1995). Interferometric PdBI and VLA observations (Tafalla \& Bachiller 1995; Gueth et al. 1998; Benedettini et al. 2007, hereafter Paper I) have mapped the brightest blue-shifted bow-shock, called B1, in (i) molecules thought to be released by dust mantles such as $\mathrm{CH}_{3} \mathrm{OH}$ and $\mathrm{NH}_{3}$; (ii) simple species, mainly sulphuretted such as CS and SO, expected to form, although less efficiently, also in the ambient gas; and (iii) $\mathrm{SiO}$, which is the typical tracer of high-speed shocks, formed after the sputtering of silicon from dust (Gusdorf et al. 2008a,b). These observations revealed a rich and clumpy structure, the clumps being located at the wall of the cavity with an arch-shape.

Recently, Arce et al. (2008) have reported the detection of complex organic molecules such as $\mathrm{HCOOCH}_{3}, \mathrm{HCOOH}$, $\mathrm{C}_{2} \mathrm{H}_{5} \mathrm{OH}$, and $\mathrm{CH}_{3} \mathrm{CN}$ towards L1157-B1. Such detections, made with the 30-m IRAM single-dish telescope, suggest that these complex molecules, usually considered as suitable hotcore tracers, can also increase in abundance due to the impact of outflows on the surrounding medium and not only due to a protostar's radiation. This finding is particularly important for $\mathrm{CH}_{3} \mathrm{CN}$ (methyl cyanide), whose emission has been extensively used to investigate the surroundings of high-mass (the so called hot-cores; e.g. Churchwell et al. 1992; Olmi et al. 1993, 1996a,b) and lower mass (hot-corinos; e.g. Cazaux et al. 2003; Bottinelli et al. 2004a,b; Bisschop et al. 2008) YSOs. In particular, $\mathrm{CH}_{3} \mathrm{CN}$ emission has been used to study the kinematics of hot-cores (Beltrán et al. 2004, 2005), revealing rotating massive toroids expected to host obscured accretion disks.

In this letter, we report for the first time the high-resolution image of $\mathrm{CH}_{3} \mathrm{CN}$ towards the L1157-B1, showing that this emission is issued from warm gas $(\sim 60-130 \mathrm{~K})$ tracing the bow structure created by the passage of shocks. 


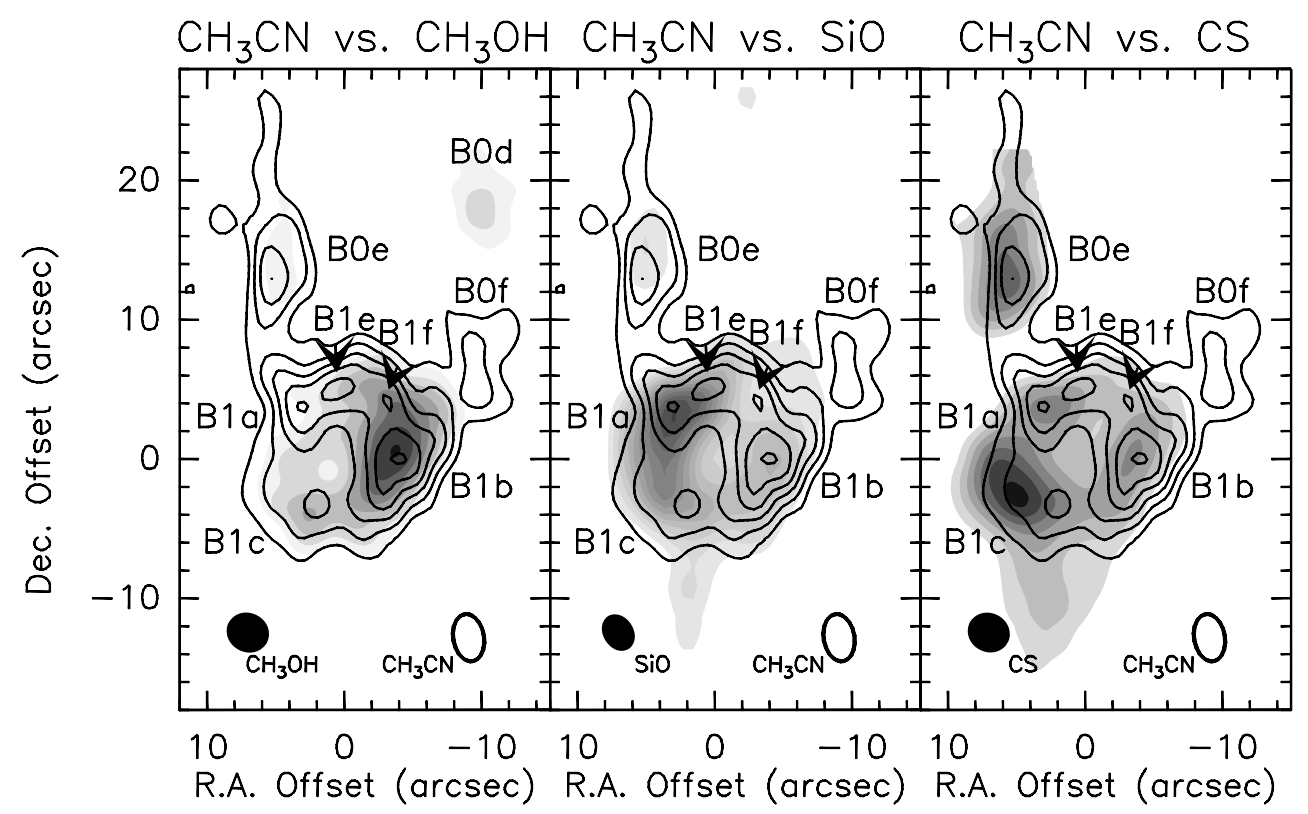

Fig. 1. Chemical differentiation in L1157-B1: the maps are centred at the following coordinates: $\alpha_{2000}=20^{\mathrm{h}} 39^{\mathrm{m}} 09^{\mathrm{s}} .5, \delta_{2000}=+68^{\circ} 01^{\prime} 10^{\prime} \cdot 0$, i.e. at $\Delta \alpha=+21^{\prime \prime} \cdot 7$ and $\Delta \delta=-64$.' 0 from the driving protostar. Overlay of the integrated intensity map of the $\mathrm{CH}_{3} \mathrm{CN}(8-7) K=0-2$ emission (contours) on the PdBI $3 \mathrm{~mm}$ emission of $\mathrm{CH}_{3} \mathrm{OH}\left(2_{1}-2_{1}\right) \mathrm{A}^{-}$(left panel), $\mathrm{SiO}(2-1)$ (middle), and $\mathrm{CS}(2-1)$ (right; grey scale, from Paper I and Gueth et al. 1998, respectively). The $\mathrm{CH}_{3} \mathrm{CN}$ contour levels range from $100 \mathrm{Jy}_{\text {beam }}^{-1}(3 \sigma)$ to $580 \mathrm{mJy}^{\mathrm{beam}}{ }^{-1}$ in steps of $80 \mathrm{mJy} \mathrm{beam}^{-1}$. The labels indicate the L1157-B1 clumps detected in $\mathrm{CH}_{3} \mathrm{CN}$ (see text), labelled following Paper I. The synthesised beams are shown in the bottom part of the panels.

\section{Observations}

The $\mathrm{CH}_{3} \mathrm{CN}$ observations of L1157-B1 were obtained in August 2008 and March 2009 with the IRAM Plateau de Bure Interferometer (PdBI) in France. Two configurations were used: the 6-element $\mathrm{C}$ configuration (baselines from $24 \mathrm{~m}$ up to $176 \mathrm{~m}$ ), and the 5-element D configuration (from $24 \mathrm{~m}$ to $97 \mathrm{~m}$ ). The $\mathrm{CH}_{3} \mathrm{CN}\left(8_{K}-7_{K}\right)$ spectral pattern at $147174.594 \mathrm{MHz}$ $(K=0)$ was observed with a $320 \mathrm{MHz}$ bandwidth and a resolution of $2.5 \mathrm{MHz}\left(\sim 5.1 \mathrm{~km} \mathrm{~s}^{-1}\right)$. Amplitude and phase were calibrated by observing $1849+670$ and $2021+614$, whereas the flux density scale was derived by observing 3C 84 and 3C 454.3, with an uncertainty of $\sim 20 \%$. Images were produced using natural weighting, and were restored with a clean beam of 3 .' $4 \times 2$ '. 3 $\left(\mathrm{PA}=10^{\circ}\right)$.

\section{3. $\mathrm{CH}_{3} \mathrm{CN}$ as tracer of shocked gas}

The contours of Fig. 1 show the map of the $\mathrm{CH}_{3} \mathrm{CN}\left(8_{K}-7_{K}\right)$ emission integrated over the $K=0-2$ components. The $\mathrm{CH}_{3} \mathrm{CN}$ structure is well in agreement with that of other molecular species observed by Tafalla \& Bachiller (1995), Gueth et al. (1998), and Paper I with the VLA and PdB interferometers, revealing a clumpy structure superimposed to the classical B1 arch-like shape seen e.g. in CO. Beside the already detected B1a, $\mathrm{B} 1 \mathrm{~b}, \mathrm{~B} 1 \mathrm{c}$ clumps, the $\mathrm{CH}_{3} \mathrm{CN}$ emission shows further emission peaks in B1, here labelled B1e and B1f. In addition, we have detected (i) the north-eastern B0e clump and (ii) a new clump, labelled B0f, towards the north-western portion of the map. In summary, the present $\mathrm{CH}_{3} \mathrm{CN}$ map clearly confirms $\mathrm{B} 1$ together with the northern B0 clumps as a unique continuous structure tracing the propagation of the large bow shock which created the large cavity called C2 (see e.g. Fig. 7 of Paper I) which was detected in CO by Gueth et al. (1996). The walls of the cavity are outlined well both by the B0e and BOf clumps and by the weaker $\mathrm{CH}_{3} \mathrm{CN}$ eastern emission between the clumps. In this context, what we have so far called clumps should not necessarily be considered isolated roundish bodies, but rather emission peaks belonging to a more elongated structure.

Figure 1 compares the $\mathrm{CH}_{3} \mathrm{CN}$ emission with those of $\mathrm{CH}_{3} \mathrm{OH}, \mathrm{SiO}$ and $\mathrm{CS}$ (Paper I; Gueth et al. 1998), mapped with similar spatial resolution. These previous observations have revealed a dichotomy suggesting an inhomogeneity in the physical conditions and/or chemical composition along B1: a group of molecules (CS, $\mathrm{SiO}$, and $\mathrm{NH}_{3}$, not shown here) peaking at the east-side and another group of species $\left(\mathrm{CH}_{3} \mathrm{OH}, \mathrm{SO}\right.$, and OCS, not shown here) peaking at the west-side of the cavity. The brightest peak of $\mathrm{CH}_{3} \mathrm{CN}$ is $\mathrm{B} 1 \mathrm{~b}$, thus well coinciding with the western $\mathrm{CH}_{3} \mathrm{OH}$ peak. Bright $\mathrm{CH}_{3} \mathrm{CN}$ emission is also found in the elongated structure composed by the B1a, B1e, and $\mathrm{B} 1 \mathrm{f}$ peaks. On the other hand, $\mathrm{CH}_{3} \mathrm{CN}$ is anticorrelated with both the apex of the bow structure as well as the so-called $\mathrm{SiO}$ and CS finger which, according to Gueth et al. (1998), could trace a magnetic precursor of the shock.

In conclusion, the present findings confirm the complexity of a region interested by the passage of a bow shock: for $\mathrm{B} 1$, in particular, $\mathrm{CH}_{3} \mathrm{CN}$ seems to introduce a third group of molecules peaking in the northern part of the B1 structure, quite apart from the effects seen in the west and east dichotomy and the $\mathrm{CS} / \mathrm{SiO}$ finger. This finding suggests temporal effects, with $\mathrm{CH}_{3} \mathrm{CN}$ mostly confined to the back part of the shock. The possible implication on the formation mechanism of $\mathrm{CH}_{3} \mathrm{CN}$ is reported in Sect. 5.

\section{The temperature structure in L1157-B1}

The temperature estimates of L1157-B1 so far obtained are based on single-dish observations of $\mathrm{CO}, \mathrm{NH}_{3}$, and $\mathrm{CH}_{3} \mathrm{CN}$ (Umemoto et al. 1999; Hirano \& Taniguchi 2001; Arce et al. 2008). The values are in the $50-170 \mathrm{~K}$ range, and they have to be considered as measurements averaged on half power beam widths $(H P B W \mathrm{~s})$ of $8^{\prime \prime}-14^{\prime \prime}(\mathrm{CO}), 72^{\prime \prime}\left(\mathrm{NH}_{3}\right)$, and 

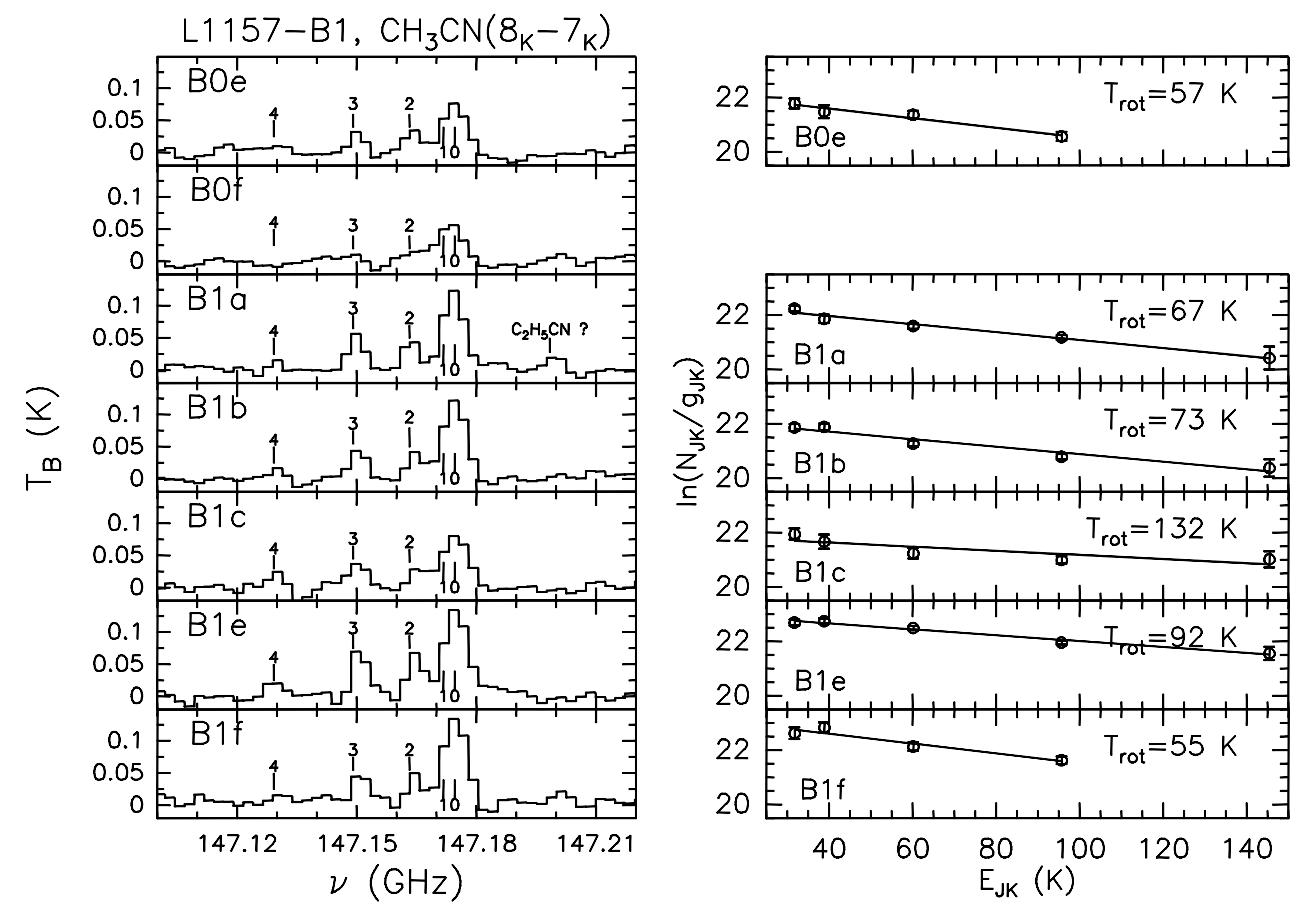

Fig. 2. Left panel: $\mathrm{CH}_{3} \mathrm{CN} J=8_{K}-7_{K}$ spectra (in $T_{\mathrm{B}}$ scale) integrated over the area of the L1157-B1 clumps. Rest frequencies of the $K$-components (from 0 to 4; see Múller et al. 2005) are pointed out by vertical bars. Note the tentative detection of the $\mathrm{C}_{2} \mathrm{H}_{5} \mathrm{~N}\left(11_{6,6}-12_{5,7}\right)(147198.4240 \mathrm{MHz}$, $\left.E_{\mathrm{u}}=68 \mathrm{~K}, S \mu^{2}=1.3 \mathrm{D}^{2}\right)$ in the B1a spectrum. Right panel: $\mathrm{CH}_{3} \mathrm{OH}$ rotation diagram with superimposed fit for all the L1157-B1 clumps but B10f, where only the $K=0$ and 1 components have been detected. The $N_{J K}, g_{J K}$, and $E_{J K}$ are, respectively, the column density and the degeneracy of the transitions reported. $E_{J K}$ is the upper level energy: $32 \mathrm{~K}(K=0), 39 \mathrm{~K}(K=1), 60 \mathrm{~K}(K=2), 96 \mathrm{~K}(K=3)$, and $143 \mathrm{~K}(K=4)$. The rotation temperatures ( $T_{\text {rot }}$, reported also in the upper-right corner of each panel) and total $\mathrm{CH}_{3} \mathrm{CN}$ column densities $\left(N_{\mathrm{CH}_{3} \mathrm{CN}}\right)$ corresponding to each clump are summarised in Table 1.

$10^{\prime \prime}\left(\mathrm{CH}_{3} \mathrm{CN}\right)$. Also the multi-lines survey of a classical shock tracer like $\mathrm{SiO}$ (Nisini et al. 2007), which shows temperatures between 100 and $300 \mathrm{~K}$, has been performed with $H P B W \mathrm{~s} \simeq$ $9^{\prime \prime}-27^{\prime \prime}$. An estimate based on a better spatial resolution (5-6") has been obtained with $\mathrm{NH}_{3}$ emission imaged at VLA by Tafalla \& Bachiller (1995), but a unique value $(80 \mathrm{~K})$ for the whole L1157-B1 structure has been reported.

Figure 2 (left panels) shows the $\mathrm{CH}_{3} \mathrm{CN}\left(8_{K}-7_{K}\right)$ spectra integrated over the area of the L1157-B1 clumps. Several $K$-components (up to $K=4$ ) are clearly detected, which have been fitted by fixing their separation in frequency in the laboratory values and forcing their line width to be identical. For a symmetric-top molecule such as $\mathrm{CH}_{3} \mathrm{CN}$, the population of one $K$-ladder relative to another is established by collisions and is characterised by a rotation temperature $\left(T_{\text {rot }}\right)$ which is approximately equal to the kinetic temperature (e.g. Loren \& Mundy 1984). We derived estimates for the rotational temperature, $T_{\text {rot }}$, and the total $\mathrm{CH}_{3} \mathrm{CN}$ column density, $\mathrm{N}_{\mathrm{CH}_{3} \mathrm{CN}}$, by means of the standard rotation diagram method. Note that the error bars refer to the line-fit uncertainty and do not take into account the absolute flux scale error (20\%), which is affecting all lines simultaneously. This means that the temperatures, identified by the slope of the fit, are not affected by the error on absolute flux. Figure 2 (right panels) shows the rotation diagrams: for the first time, it has been possible to estimate a temperature for several L1157-B1 clumps, thus allowing to derive a temperature distribution covering the whole B1 structure. The rotational temperatures are in the $57-132 \mathrm{~K}$ range. The higher $T_{\text {rot }}$ has been measured at the B1c position, near the apex of the bow structure and the $\mathrm{CS} / \mathrm{SiO}$ finger, which should lie along the jet direction and represent the magnetic precursor of the shock (Gueth et al. 1998). In addition, the temperature estimates are lower at the edge of the shock ( $67 \mathrm{~K}$ for $\mathrm{B} 1 \mathrm{a}$ and $73 \mathrm{~K}$ for $\mathrm{B} 1 \mathrm{~b}$ ). Also the B0e and B1f clumps, where only $4 K$-components have been detected (up to $K=3$ ), show a relatively low temperature: $57 \mathrm{~K}$ and $55 \mathrm{~K}$, respectively; note that the upper limits to the $K=4$ integrated intensity are consistent with a temperature less than $60 \mathrm{~K}$, confirming the result. On the other hand, $T_{\text {rot }}$ is higher in B1e $(92 \mathrm{~K})$, which is located on the jet axis, and that is where the highest blue-shifted velocities $\left(\left|V-V_{\text {sys }} \geq 10 \mathrm{~km} \mathrm{~s}^{-1}\right|\right)$ have been observed (Gueth et al. 1998; Paper I). These findings seem to confirm the presence of a bow-shock with a temperature gradient decreasing with distance from the apex, supporting the association of $\mathrm{CH}_{3} \mathrm{CN}$ with shocked gas.

Interestingly, a fit of the B1c rotation diagram without the $K=4$ component leads to $T_{\text {rot }}=72 \mathrm{~K}$ (instead of $132 \mathrm{~K}$ ). By excluding the $K=4$ line also the B1b temperature decreases from $73 \mathrm{~K}$ to $55 \mathrm{~K}$ and that of B1e from $92 \mathrm{~K}$ to $81 \mathrm{~K}$; on the opposite the $\mathrm{B} 1 \mathrm{a}$ rotation diagram keeps the same solution $(67 \mathrm{~K})$ with or without the $K=4$ emission. These results clearly illustrate the importance of probing temperatures in regions associated with high temperatures, such as shocked regions, by using high excitation transitions. These results suggest two gas components with different temperatures and could be related to the $\mathrm{SiO}$ results obtained by Nisini et al. (2007), which show evidence for velocity components having different excitation conditions, with the denser and/or warmer gas associated with higher speeds: further high spectral resolution observations would be instructive.

\section{On the origin of $\mathrm{CH}_{3} \mathrm{CN}$ in bow-shocks}

The origin of $\mathrm{CH}_{3} \mathrm{CN}$ molecules in star forming regions is still a matter of debate (see e.g. Bottinelli et al. 2007; and Bisschop et al. 2008, for detailed reviews). One possibility is that they 
Table 1. The L1157-B1 clumps: $\mathrm{CH}_{3} \mathrm{CN}$ rotational temperatures, and $\mathrm{CH}_{3} \mathrm{CN}$ and $\mathrm{CH}_{3} \mathrm{OH}$ column densities.

\begin{tabular}{lcccc}
\hline \hline Clump & $\begin{array}{c}T_{\text {rot }} \\
(\mathrm{K})\end{array}$ & $\begin{array}{c}N_{\mathrm{CH}_{3} \mathrm{CN}} \\
\left(\mathrm{cm}^{-2}\right)\end{array}$ & $\begin{array}{c}N_{\mathrm{CH}_{3} \mathrm{OH}}{ }^{a} \\
\left(\mathrm{~cm}^{-2}\right)\end{array}$ & $\begin{array}{c}{\left[\mathrm{CH}_{3} \mathrm{CN}\right] /\left[\mathrm{CH}_{3} \mathrm{OH}\right]} \\
\left(10^{-3}\right)\end{array}$ \\
\hline B0e & 57 & $8 \times 10^{12}$ & $6 \times 10^{15}$ & 1.3 \\
B0f & $-b$ & $-{ }^{b}$ & $-^{a}$ & - \\
B1a & 67 & $1 \times 10^{13}$ & $4 \times 10^{16}$ & 0.3 \\
B1b & 73 & $1 \times 10^{13}$ & $5 \times 10^{16}$ & 0.2 \\
B1c & 132 & $2 \times 10^{13}$ & $4 \times 10^{16}$ & 0.5 \\
B1e & 92 & $4 \times 10^{13}$ & $-^{a}$ & - \\
B1f & 55 & $2 \times 10^{13}$ & $-^{a}$ & - \\
\hline
\end{tabular}

${ }^{a}$ From Paper I: B0f, B1e, and B1f not identified; ${ }^{b}$ only the $K=0$ and 1 components are clearly detected.

form on the surface of grains from reactions of $\mathrm{CH}_{3}$ with $\mathrm{CN}$ and are then injected in the gas phase, together with species such as $\mathrm{H}_{2} \mathrm{O}, \mathrm{H}_{2} \mathrm{CO}$, and $\mathrm{CH}_{3} \mathrm{OH}$, once the icy grain mantles evaporate. Otherwise, $\mathrm{CH}_{3} \mathrm{CN}$ may belong to the so-called second generation species, i.e. the molecules formed in the warm gas-phase chemically enriched after the mantle evaporation; in this case $\mathrm{CH}_{3} \mathrm{CN}$ would form from $\mathrm{HCN}$ with $\mathrm{CH}_{3}{ }^{+}$. Both scenarios need an increase of temperature, due to stellar radiation in case of hot-core and hot-corinos or the passage of a shock in the present case, confirmed by the high $T_{\text {rot }}$ measured here. As chemical models for hot-cores and corinos have shown, the warm-up phase plays a major role in both scenarios (Viti et al. 2004; Garrod \& Herbst 2006). Under the assumption of the more realistic gradual warm-up of temperatures instead of an instantaneous jump from 10 to several hundred kelvins, then (i) the changes in the chemical evolution of each species due to differential desorption are important and several species, including $\mathrm{CH}_{3} \mathrm{OH}$ and $\mathrm{CH}_{3} \mathrm{CN}$ show a strong time dependence (Viti et al. 2004) and (ii) gas-phase and grain-surface reactions are strongly coupled during the warm-up phase (Garrod \& Herbst 2006). For example, $\mathrm{CN}$ and $\mathrm{CH}_{3}$ may become more mobile on the grains enhancing the production of $\mathrm{CH}_{3} \mathrm{CN}$. On the other hand, if instead the evaporation is caused by the passage of the shock, it will be effectively instantaneous due to sputtering; hence only surface reactions during the freeze-out process and gas phase reactions would be relevant for the production of $\mathrm{CH}_{3} \mathrm{CN}$.

The $N_{\mathrm{CH}_{3} \mathrm{CN}}$ column densities, reported in Table 1, are in the $8-40 \times 10^{12} \mathrm{~cm}^{-2}$ range. These values are larger than those given by Arce et al. (2008), $1 \times 10^{12} \mathrm{~cm}^{-2}$ : this is not surprising, since their estimate is based on a single-dish spectrum producing a $\mathrm{CH}_{3} \mathrm{CN}$ brightness and temperature averaged with a $H P B W \sim$ $10^{\prime \prime}$, affected by beam dilution due to the clumpy structure of the emission. We derived the abundance ratio of $\mathrm{CH}_{3} \mathrm{CN}$ with respect to $\mathrm{CH}_{3} \mathrm{OH}$, which is a well known abundant molecule injected in the gas phase from warmed dust mantles. By using the $N_{\mathrm{CH}_{3} \mathrm{OH}}$ values calculated in Paper I, we obtain $\left[\mathrm{CH}_{3} \mathrm{CN}\right] /\left[\mathrm{CH}_{3} \mathrm{OH}\right]$ abundance ratios in the $0.2-1.3 \times 10^{-3}$ range, depending on the clump. Even with higher $N_{\mathrm{CH}_{3} \mathrm{CN}}$ values with respect to Arce et al. (2008), the $\mathrm{CH}_{3} \mathrm{CN}$-to- $\mathrm{CH}_{3} \mathrm{OH}$ ratios are still on average smaller with respect to those found in hot-corinos (0.3-1 $\times 10^{-1}$; Bottinelli et al. 2007; Bisschop et al. 2008), as well as those found in massive hot-cores $\left(\sim 10^{-2}-10^{-1}\right.$; Bisschop et al. 2007). This difference suggests that asignificant amount of $\mathrm{CH}_{3} \mathrm{CN}$ may form in the gas phase and that in the shocked L1157-B1 regions the $\mathrm{CH}_{3} \mathrm{CN}$ abundance has not yet reached its maximum, given that the age of the warm gas in L1157-B1 has been estimated to be less than $2000 \mathrm{yr}$ (Arce et al. 2008). This possibility is supported by the relative $\mathrm{CH}_{3} \mathrm{CN}$ weakness of the apex region of the shock compared to the rear of the bow. The $\left[\mathrm{CH}_{3} \mathrm{CN}\right] /\left[\mathrm{CH}_{3} \mathrm{OH}\right]$ ratio, however, is found to vary by several orders of magnitude among hotcores/corinos, depending on their physical properties as well as their evolutionary stage.

\section{Conclusions}

The present high spatial resolution map shows that $\mathrm{CH}_{3} \mathrm{CN}$ can be efficiently produced in regions far from the protostar and affected by the passage of a shock wave. Thanks to the analysis of the $\mathrm{CH}_{3} \mathrm{CN}\left(8_{K}-7_{K}\right)$ spectral pattern, we obtained for the first time estimates of the temperature at six different positions covering the whole B1 structure. This is essential for the correct analysis of the forthcoming spectral survey of L1157-B1 that will be carried out by the ESA Herschel satellite in Guaranteed Time. The survey will cover the $500-2000 \mathrm{GHz}$ spectral window to obtain an accurate census of the molecular content in star forming regions. The knowledge of the physical properties of the L1157-B1 clumps, used as proto-type of shocked regions, is needed to interpret the Herschel spectra, which will be obtained with a $10^{\prime \prime}-40^{\prime \prime}$ spatial resolution.

Acknowledgements. We thank R. Cesaroni for helpful discussion.

\section{References}

Arce, H. G., Santiago-García, J., Jörgensen, J. K., Tafalla, M., \& Bachiller, R. 2008, ApJ, 681, L21

Bachiller, R., \& Peréz Gutiérrez, M. 1999, ApJ, 487, L93

Bachiller, R., Peréz Gutiérrez, M., Kumar, M. S. N., \& Tafalla, M. 2001, A\&A, 372,899

Benedettini, M., Viti, S., Codella, C., et al. 2007, MNRAS, 381, 1127 (Paper I) Beltrán, M. T., Cesaroni, R., Neri, R., et al. 2004, ApJ, 601, L187

Beltrán, M. T., Cesaroni, R., Neri, R., et al. 2005, A\&A, 435, 901

Bisschop, S. E., Jörgensen, J. K., van Dishoeck, E. F., \& de Wachter, E. B. M. 2007, A\&A, 465, 913

Bisschop, S. E., Jörgensen, J. K., Bourke, T. L., Bottinelli, S., \& van Dishoeck, E. F. 2008, A\&A, 488, 959

Bottinelli, S., Ceccarelli, C., Lefloch, B., et al. 2004a, ApJ, 615, 354

Bottinelli, S., Ceccarelli, C., Neri, R., et al. 2004b, ApJ, 617, L69

Bottinelli, S., Ceccarelli, C., Williams, J. P., \& lefloch, B. 2007, A\&A, 463, 601

Cazaux, S., Tielens, A. G. G. M., Ceccarelli, C., et al. 2003, ApJ, 593, L51

Churchwell, E., Walmsley, C. M., \& Wood, D. O. S. 1992, A\&A, 253, 541

Davis, C. J., \& Eislöffel, J. 1995, A\&A, 300, 851

Garay, G., Köhnenkamp, I., Bourke, T. L., Rodríguez, L. F., \& Lehtinen, K. K. 1998, ApJ, 509, 768

Garrod, R. T., \& Herbst, E. 2006, A\&A, 457, 927

Gueth, F., Guilloteau, S., \& Bachiller, R. 1996, A\&A, 307, 891

Gueth, F., Guilloteau, S., \& Bachiller, R. 1998, A\&A, 333, 287

Gusdorf, A., Cabrit, S., Flower, D. R., \& Pineau Des Forêts, G. 2008a, A\&A, 482,809

Gusdorf, A., Pineau Des Forêts, G., Cabrit, S., \& Flower, D. R. 2008b, A\&A, 490, 695

Hirano, N., \& Taniguchi, Y. 2001, ApJ, 550, L219

Jörgensen, J. K., Bourke, T. L., \& Myers, P. C. 2007, ApJ, 659, 479

Loren, R. B., \& Mundy, L. G. 1984, ApJ, 286, 232

Müller, H. S. P., Schlóder, F., Stutzki, J., \& Winnewisser, G. 2005, J. Mol. Struct., 742,215

Nisini, B., Codella, C., Giannini, T., et al. 2007, A\&A, 462, 163

Olmi, L., Cesaroni, R., \& Walmsley, C. M. 1993, A\&A, 276, 489

Olmi, L., Cesaroni, R., \& Walmsley, C. M. 1996a, A\&A, 307, 599

Olmi, L., Cesaroni, R., Neri, R., \& Walmsley, C. M. 1996b, A\&A, 315, 565 Tafalla, M., \& Bachiller, R. 1995, ApJ, 443, L37

Ummoto, T., Mikami, H., Yamamoto, S., \& Hirano, N. 1999, ApJ, 525, L105

van Dishoeck, E. F., \& Blake, G. A. 1998, ARA\&A, 36, 317

Viti, S., Collings, M. P., Dever, J. W., McCoustra, M. R. S., \& Williams, D. A. 2004, MNRAS, 354, 1141 